\title{
A New Ordering Parameter for Blazar SEDs
}

\author{
Michael Zacharias* and Reinhard Schlickeiser \\ Institut für theoretische Physik IV, Ruhr-Universität Bochum, Germany \\ E-mail: mz@tp4.rub.de, rsch@tp4.rub.de
}

\begin{abstract}
Compton dominated blazars exhibit non-linear cooling, if the emission of the high-energy peak is attributed to synchrotron self-Compton (SSC) radiation. This leads to some striking features in the emerging spectral energy distribution (SED). We report of a recent theoretical work (Zacharias \& Schlickeiser 2011), where we analyzed in detail the consequences of the non-linear cooling. Two obvious results emerge: Firstly, depending on the initial cooling being either linear or non-linear, the dominating peak is either the synchrotron or the SSC peak. Secondly, for initial non-linear cooling the synchrotron peak exhibits a unique broken power-law, which can be directly attributed to the non-linear cooling regardless of the underlying electron distribution. In order to prove the capability of the approach, we apply our model to large flares of the blazars 3C 279 and 3C 454.3. We achieve rather good fits with reasonable parameters. The results are very promising, and we argue that SSC cooling should be considered for future modeling attempts of blazars exhibiting a dominating inverse Compton peak.
\end{abstract}

AGN Physics in the CTA Era - AGN2011,

May 16-17, 2011

Toulouse, France

${ }^{*}$ Speaker. 


\section{Introduction}

This proceeding is a summary of a recent work (Zacharias \& Schlickeiser 2011) about the effects of SSC cooled electrons on the spectra of blazars. For all the details about the calculations and for extensive discussions of the approach and the results we refer to that paper.

It is widely accepted that the two-component structure of blazars is due to synchrotron and inverse Compton (IC) processes of highly relativistic electrons moving randomly in a plasma blob in the jet of a supermassive black hole in the heart of an active galactic nucleus (AGN). The lowenergy component is attributed to synchrotron radiation, while the high-energy component results from IC collisions of the electrons with a photon field (Böttcher 2007). These photons either come from an external source, like the accretion disk (Dermer and Schlickeiser 1993), the broad line regions (Sikora et al. 1994) or the dusty torus (Blazejowski et al. 2000), or from the inevitably present synchrotron emission of the electrons (Jones et al. 1974). The latter is referred to as the synchrotron self-Compton (SSC) process, and it has been shown by Schlickeiser (2009) that this interaction leads to non-linear cooling of the electrons, since the scattered radiation field needs to be produced by the same electrons first.

This energy loss is described by the equation

$$
|\dot{\gamma}(\gamma, t)|_{\mathrm{SST}}=A_{0} \gamma^{2} \int_{0}^{\infty} d \tilde{\gamma} \tilde{\gamma}^{2} n(\tilde{\gamma}, t), A_{0}=\frac{3 \sigma_{T} c_{1} P_{0} R \varepsilon_{0}^{2}}{m c^{2}},
$$

while the linear synchrotron loss of an electron in a constant magnetic field with strength $B=b$ Gauss is

$$
|\dot{\gamma}(\gamma)|_{S}=D_{0} \gamma^{2}, \quad D_{0}=\frac{4}{3} \frac{c \sigma_{T}}{m c^{2}} U_{B}=1.29 \cdot 10^{-9} b^{2} \mathrm{~s}^{-1} .
$$

Here $\gamma$ refers to the electron Lorentz factor, $n(\gamma, t)$ describes the electron distribution function, $P_{0}=3.2 \cdot 10^{12} \mathrm{eV}^{-1} \mathrm{~s}^{-1}, \varepsilon_{0}=1.16 \cdot 10^{-8} \mathrm{beV}, R$ is the radius of the spherical source, $m c^{2}$ denotes the electron rest energy, $\sigma_{T}=6.65 \cdot 10^{-25} \mathrm{~cm}^{2}$ is the Thomson cross section, and $c_{1}=0.684$.

According to Schlickeiser, Böttcher and Menzler (2010, hereafter SBM) the flux of the respective peaks is directly correlated with the cooling terms. This implies that the ratio of the initial cooling terms also reflects the ratio of the peak fluxes, a term sometimes referred to as the Compton dominance. We express this ratio by the ordering parameter

$$
\alpha^{2}=\frac{|\dot{\gamma}(\gamma, t=0)|_{\text {SST }}}{|\dot{\gamma}(\gamma)|_{S}} .
$$

Thus, if the cooling is initially linear, that is to say $\alpha \ll 1$, we expect a dominance of the synchrotron peak. In the other case of an initial non-linear cooling with $\alpha \gg 1$ the IC peak should exhibit a higher flux.

It has been shown by SBM that, although the non-linear cooling acts quicker than the linear cooling, the cooling will eventually become linear after some time. This is taken into account by combining both cooling terms to a single one:

$$
|\dot{\gamma}(\gamma, t)|=\gamma^{2}\left(D_{0}+A_{0} \int_{0}^{\infty} d \tilde{\gamma} \tilde{\gamma}^{2} n(\tilde{\gamma}, t)\right) .
$$

This term has some important consequences for the resulting electron distribution and the emerging spectral energy distribution (SED). We will discuss them in section 2 and 3, respectively. Applications to the blazars 3C 279 and 3C 454.3 are given in section 4 . 


\section{Electron distribution function}

The electron distribution $n(\gamma, t)$ obeys the differential equation (Kardashew 1962)

$$
\frac{\partial n(\gamma, t)}{\partial t}-\frac{\partial}{\partial \gamma}\left[\gamma^{2}\left(D_{0}+A_{0} \int_{0}^{\infty} d \gamma \gamma^{2} n(\gamma, t)\right) n(\gamma, t)\right]=Q_{0} \delta\left(\gamma-\gamma_{0}\right) \delta(t)
$$

where $Q_{0}$ is the injection constant and $\gamma_{0}$ is the initial Lorentz factor of the electrons.

The solution depends on $\alpha=\frac{\gamma_{0}}{\gamma_{B}}$, where the characteristic electron Lorentz-factor $\gamma_{B}=\left(\frac{D_{0}}{A_{0} Q_{0}}\right)^{1 / 2}$ reflects the injection conditions of the relativistic electrons in a spherical homogeneous source. Obviously, the higher the electron density in the source the higher the probability of non-linear cooling.

The electron distribution function then becomes for $\alpha \ll 1$

$$
n(\gamma, t, \alpha \ll 1)=Q_{0} H\left[\gamma_{0}-\gamma\right] \delta\left(\gamma-\frac{\gamma_{0}}{1+D_{0} \gamma_{0} t}\right),
$$

which is solely determined by the linear synchrotron cooling.

For $\alpha \gg 1$ the solution is divided into two parts for early and late times. For early times one finds

$$
n\left(\gamma, \gamma_{0}, t \leq t_{c}, \alpha \gg 1\right)=Q_{0} H\left[\gamma_{0}-\gamma\right] H\left[t_{c}-t\right] \delta\left(\gamma-\frac{\gamma_{0}}{\left(1+3 A_{0} Q_{0} \gamma_{0}^{3} t\right)^{1 / 3}}\right),
$$

reflecting the nonlinear cooling. For later times

$$
n\left(\gamma, \gamma_{0}, t \geq t_{c}, \alpha \gg 1\right)=Q_{0} H\left[\gamma_{B}-\gamma\right] H\left[t-t_{c}\right] \delta\left(\gamma-\frac{\gamma_{B}}{\frac{1+2 \alpha^{3}}{3 \alpha^{3}}+D_{0} \gamma_{B} t}\right),
$$

which is a modified linear solution. The critical time $t_{c}=\frac{1}{3 \gamma_{B} D_{0}}$.

The monochromatic injection term for the electrons is well justified by the work of Zacharias and Schlickeiser (2010). They made a similar calculation, but with an injection of a power-law with an electron spectral index $s$. They found that once the cooling takes over the electron distribution is severely quenched into a near-monochromatic function. Comparing their approach with a pure monochromatic one like in SBM, one finds for the emerging synchrotron spectrum that the main difference is only for the high-energetic part beyond a certain threshold. While the spectrum of monochromatic electrons exhibits an exponential cut-off, the spectrum of power-law distributed electrons shows also a power-law depending on $s$. However, below said threshold the emerging radiation spectra are indistinguishable. We conclude that the monoenergetic approach is a late time limit for any extended injection and most of the important spectral features are still recovered.

\section{Spectral energy distribution}

Zacharias and Schlickeiser (2011) calculated the SEDs for both cases of $\alpha$. For very high energetic electrons the Klein-Nishina limit needs to be taken into account, which results in a further division.

The SEDs are derived by integrating the electron distribution, which results first in the timedependent intensity spectrum. A time-integration then leads to the fluence spectrum $F(v)$. Multiplying this with the frequency $v$ gives the SED $D(v)=v F(v)$. Transforming into the system of 
rest of the host galaxy, which is for low red-shifts almost the observer's frame, the SED becomes $D^{\prime}\left(v^{\prime}\right)=\delta^{2} D\left(v^{\prime} / \delta\right)$, where $\delta=\left[\Gamma\left(1-\beta_{\Gamma} \cos \theta_{o b s}\right)\right]^{-1}$ is the Doppler-factor and primed quantities are measured in the observer's frame.

The SEDs depend only on four parameters, namely the ordering parameter $\alpha$, the initial electron Lorentz factor $\gamma_{0}=10^{4} \gamma_{4}$, the Doppler-factor $\delta$, and the magnetic field $B=b$ Gauss.

\subsection{Synchrotron dominance}

This case is characterized by $\alpha \ll 1$. The SEDs for the Thomson- $\left(\gamma_{4} \ll(0.136 \cdot b)^{-1 / 3}\right)$ and the Klein-Nishina-limit $\left(\gamma_{4} \gg(0.136 \cdot b)^{-1 / 3}\right)$ are shown in Figs. 1 and 2, respectively.
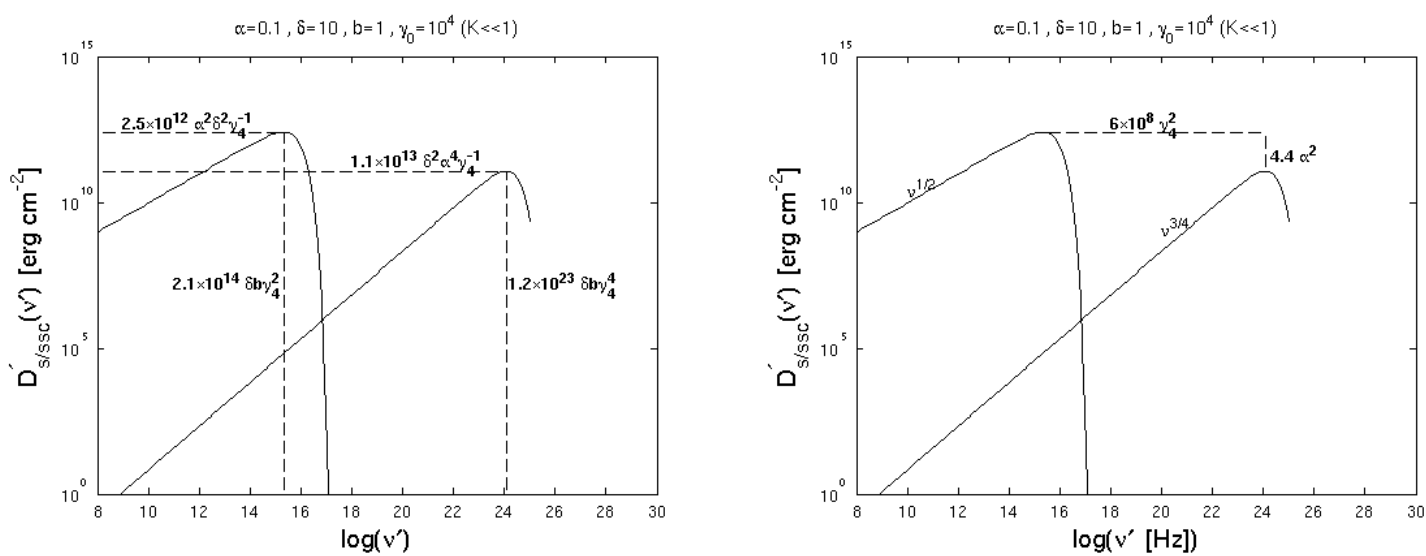

Figure 1: SED for $\alpha \ll 1$ in the Thomson-limit $\left(\gamma_{4} \ll(0.136 \cdot b)^{-1 / 3}\right)$. The parameters are given at the top. Left: Indicated are the peak values (horizontal line) and the peak frequencies (vertical lines). Right: Indicated are the ratio of the peak values (vertical line) and of the peak frequencies (horizontal line), as well as the power-laws.
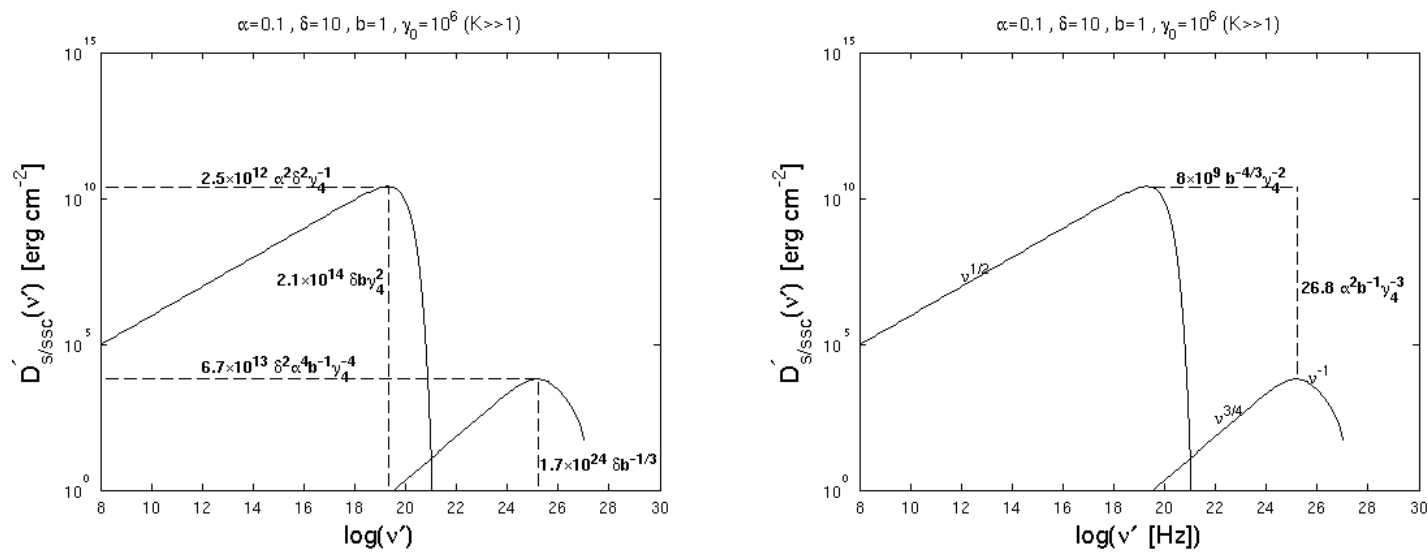

Figure 2: SED for $\alpha \ll 1$ in the Klein-Nishina-limit $\left(\gamma_{4} \gg(0.136 \cdot b)^{-1 / 3}\right)$. The parameters are given at the top. Left: Indicated are the peak values (horizontal line) and the peak frequencies (vertical lines). Right: Indicated are the ratio of the peak values (vertical line) and of the peak frequencies (horizontal line), as well as the power-laws. 
One can clearly see the dominance of the synchrotron peak over the inverse Compton peak, just as we expected, and also the relatively simple structure of the synchrotron peak exhibiting only a single power-law $v^{\prime 1 / 2}$ followed by an exponential cut-off.

\subsection{SSC dominance}

In Figs. 3-5 we plot the results for $\alpha \gg 1$ in the Thomson- $\left(\gamma_{4} \ll(0.136 \cdot b)^{-1 / 3}\right)$, mild Klein-Nishina- $\left(\gamma_{4} \ll \alpha(0.136 \cdot b)^{-1 / 3}\right)$ and extreme Klein-Nishina-limit $\left(\gamma_{4} \gg \alpha(0.136 \cdot b)^{-1 / 3}\right)$, respectively.
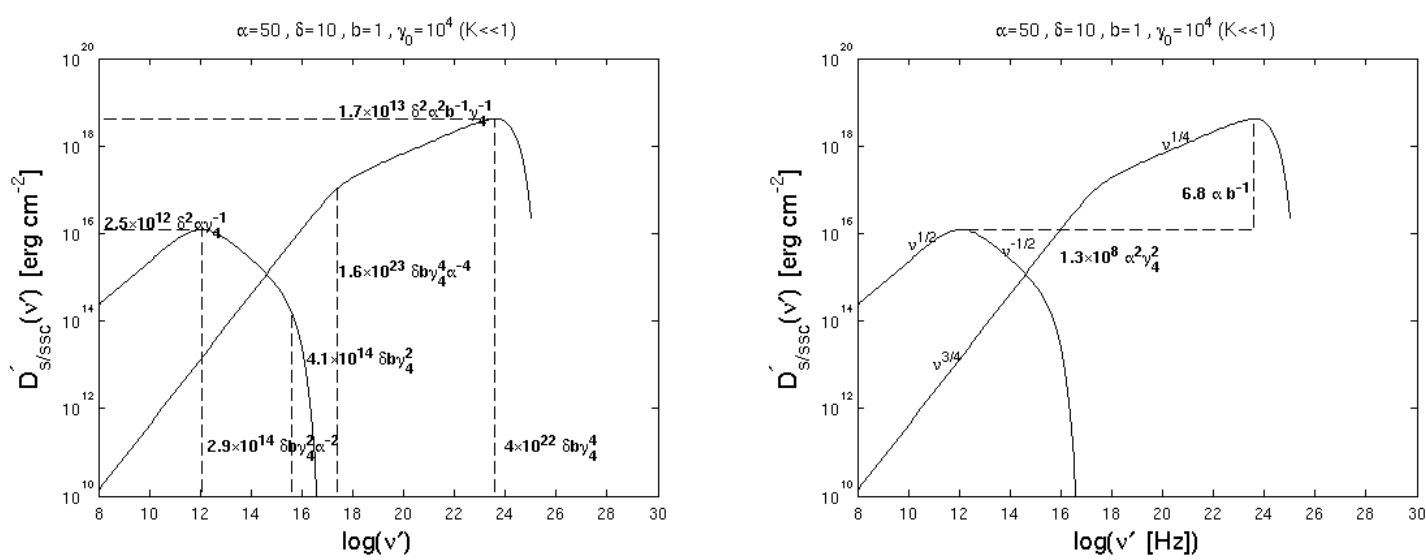

Figure 3: SED for $\alpha \gg 1$ in the Thomson-limit $\left(\gamma_{4} \ll(0.136 \cdot b)^{-1 / 3}\right)$. The parameters are given at the top. Left: Indicated are the peak values (horizontal line) and the peak frequencies (vertical lines). Right: Indicated are the ratio of the peak values (vertical line) and of the peak frequencies (horizontal line), as well as the power-laws.
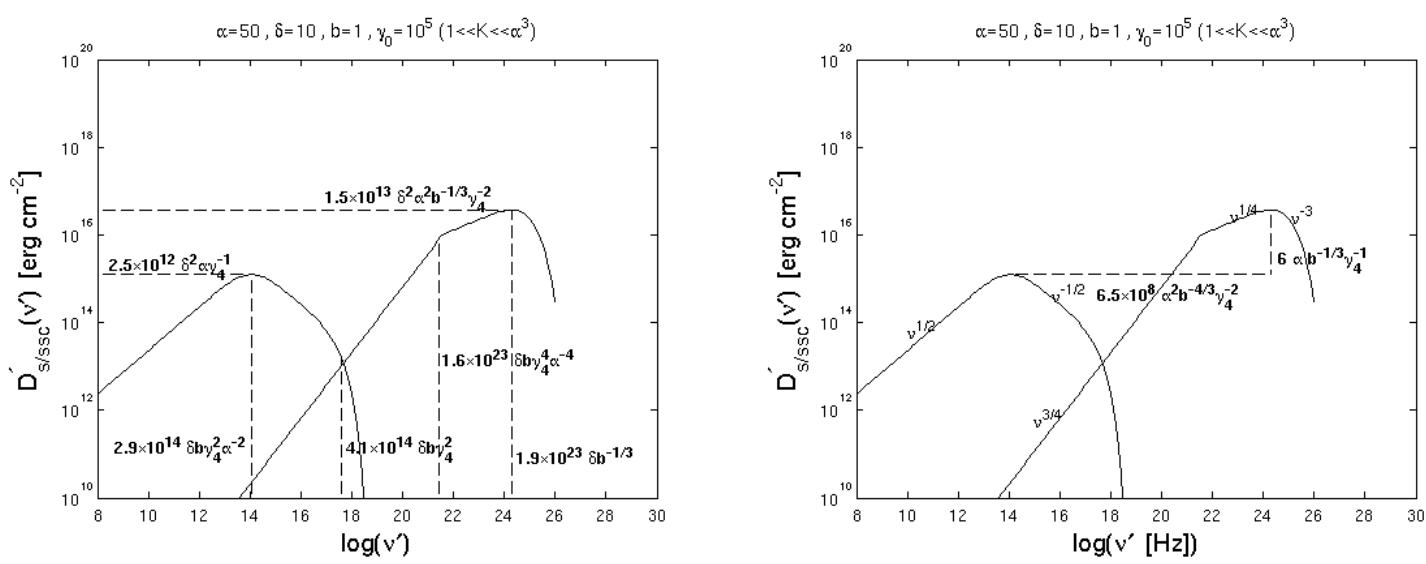

Figure 4: SED for $\alpha \gg 1$ in the mild Klein-Nishina-limit $\left(\gamma_{4} \ll \alpha(0.136 \cdot b)^{-1 / 3}\right)$. The parameters are given at the top. Left: Indicated are the peak values (horizontal line) and the peak frequencies (vertical lines). Right: Indicated are the ratio of the peak values (vertical line) and of the peak frequencies (horizontal line), as well as the power-laws.

The first obvious difference to the case $\alpha \ll 1$ is the dominance of the SSC peak over the synchrotron peak, also in agreement with our expectations. Another very important feature is the 

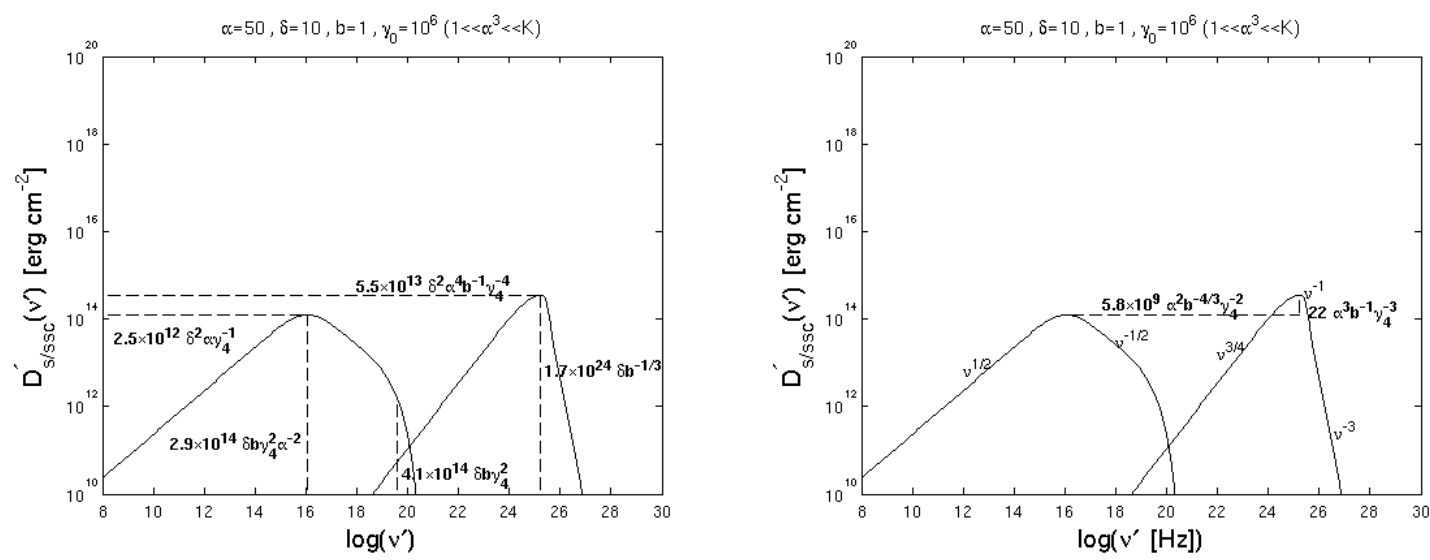

Figure 5: SED for $\alpha \gg 1$ in the extreme Klein-Nishina-limit $\left(\gamma_{4} \gg \alpha(0.136 \cdot b)^{-1 / 3}\right)$. The parameters are given at the top. Left: Indicated are the peak values (horizontal line) and the peak frequencies (vertical lines). Right: Indicated are the ratio of the peak values (vertical line) and of the peak frequencies (horizontal line), as well as the power-laws.

broken power-law of the synchrotron peak, which is a unique feature of the non-linear cooling, and independent of the injection spectrum of the electrons (c.f. Zacharias and Schlickeiser (2010)).

\section{Illustrative examples: The cases of $3 \mathrm{C} 279$ and $3 \mathrm{C} 454.3$}

In this section we intend to show the capabilities of our model by applying it to flares of $3 \mathrm{C}$ 279 and 3 C 454.3, but without any statistical analysis.
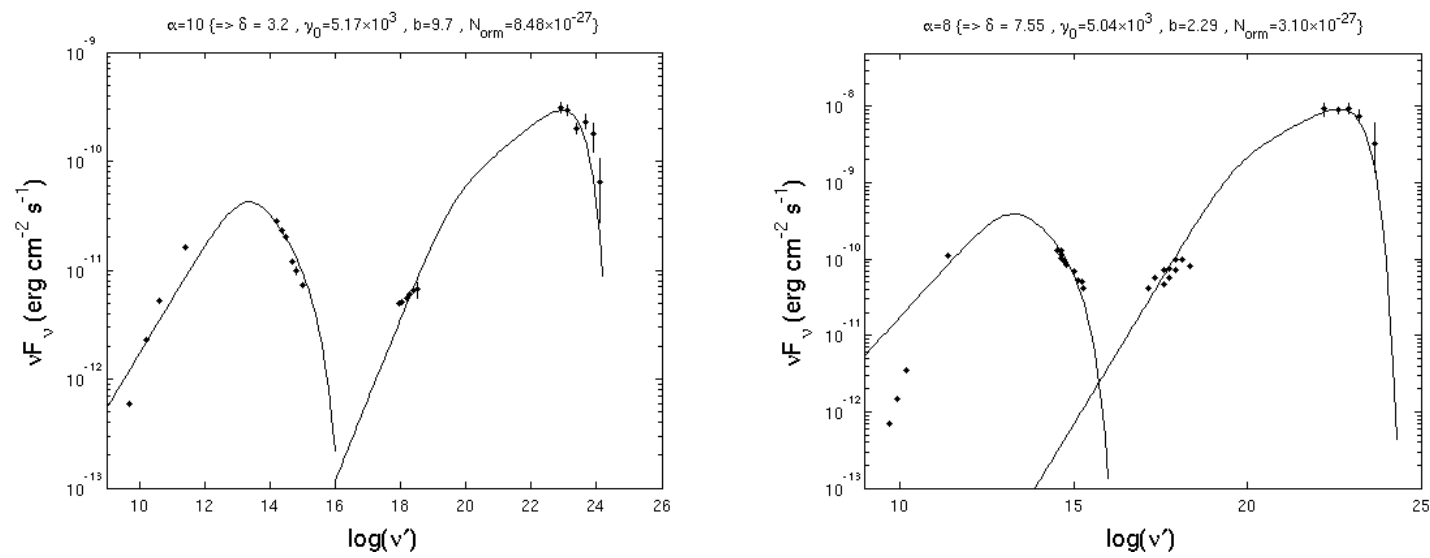

Figure 6: Left: The outburst of 3C 279 from February 2009. Right: The outburst of 3C 454.3 from November 2010. In both pictures the data is overlaid with the model in the case $\alpha \gg 1$ in the Thomson limit. The respective parameters are indicated at the top.

In the left panel of Fig. 6 we show the data of a major outburst of the blazar 3C 279 from February 2009 (Abdo et al. 2010), while the right panel shows a similarly significant flare of 3C 454.3 from November 2010 (Vercellone et al. 2011). In both cases the SSC peak clearly dominates the synchrotron peak, thus we chose the model with $\alpha \gg 1$ in the Thomson-limit to model each 
spectrum. As one can see, we can match the data relatively well with the parameters $\alpha=10$, $\delta=3.2, b=9.7$, and $\gamma_{0}=5.17 \cdot 10^{3}$ for $3 \mathrm{C} 279$, and $\alpha=8, \delta=7.55, b=2.29$, and $\gamma_{0}=5.04 \cdot 10^{3}$ for $3 \mathrm{C}$ 454.3. The remaining parameter $N_{\text {orm }}$ is used to normalize the spectrum. It mainly depends on the (known) red-shift and the radius of the source.

The parameters are very reasonable. Especially, the low Doppler- and Lorentz-factors are striking, although they may lead to internal absorption of $\gamma$-rays in the source. We intend to address this problem in future work.

\section{Discussion and conclusions}

We have presented the result on a recent study on the SEDs of blazars which exhibit a combined linear and non-linear cooling (Zacharias \& Schlickeiser 2011). The SEDs depend critically on the ordering parameter $\alpha$, which describes the type of initial cooling. For $\alpha \ll 1$ the cooling is entirely linear and the well known results are obtained, with a dominance of the synchrotron peak over the SSC peak. For $\alpha \gg 1$ the cooling is initially non-linear and some striking new features emerge in the SED. First of all, the SSC peak now dominates over the synchrotron peak. Secondly, the synchrotron peak shows a specific broken power-law, which can be directly attributed to the non-linear cooling. These two points should give observers a clear indication if the source has cooled under this non-linear process.

In order to show the capabilities of our approach, we modeled the SEDs of 3C 279 and 3C 454.3 for large outbursts in February 2009 and November 2010, respectively. With very reasonable values of the free parameters we achieved very good fits of the data without the need for external photon fields.

We argue that this is clear evidence that the sources cooled via the SSC channel, and that this scenario should be considered by observers for future modeling attempts of blazar flares.

\section{References}

[1] Abdo A. A., et al., 2010, Nature 463, 919

[2] Blazejowski M., et al., 2000, ApJ 545, 107

[3] Böttcher M., 2007, Astroph. \& Space Sci. 309, 95

[4] Dermer C. D., Schlickeiser R., 1993, ApJ 416, 458

[5] Jones T.W., O’Dell S.L., Stein W.A., 1974, ApJ 188, 353

[6] Schlickeiser R., 2009, MNRAS 398, 1483

[7] Schlickeiser R., Böttcher M., Menzler U., 2010, A\&A 519, A9 (SBM)

[8] Sikora M., Begelman M. C., Rees M. J., 1994, ApJ 421, 153

[9] Vercellone S., et al., 2011, ApJL 736, L38

[10] Zacharias M., Schlickeiser R., 2010, A\&A 524, A31

[11] Zacharias M., Schlickeiser R., 2011, MNRAS in press, (preprint arxiv:1110.2904) 